\title{
Conditional inactivation of Miwi2 reveals that MIWI2 is only essential for prospermatogonial development in mice
}

\author{
J Bao ${ }^{1}$, Y Zhang ${ }^{1}$, AS Schuster ${ }^{1}$, N Ortogero ${ }^{1}$, EE Nilsson ${ }^{2}$, MK Skinner ${ }^{2}$ and W Yan ${ }^{\star, 1}$
}

The PIWI-piRNA pathway serves as a critical defense mechanism through which the genome of the male germline is protected from invasion by transposable elements (TEs). MIWI2/PIWIL4, a member of the murine PIWI subclade of the Argonaute family, has been shown to be expressed in primordial germ cells (PGCs) and prospermatogonia in fetal and prepubertal testes. Global inactivation of Miwi2 leads to male sterility due to an early meiotic arrest, which correlates with retrotransposon desuppression. However, it remains unclear whether MIWI2 functions beyond the PGC stage and whether MIWI2 has a role beyond TE suppression during male germ line development. Through conditional inactivation of Miwi2, we demonstrate herein that MIWI2 function is restricted to a narrow time window during male PGC reprograming and that Miwi2 is dispensable for postnatal male germline development and testicular function in mice. Moreover, persistent activation of LINE1 and IAP retrotransposons caused by Miwi2 inactivation is compatible with mitotic cell cycle progression of spermatogonia during the first wave of spermatogenesis, but can cause zygotene to pachytene arrest in early meiosis due to multiple defects including enhanced DNA double-strand breaks, aberrant histone modifications and altered mRNA transcriptome. Our data not only validate those from global Miwi2 KO studies, but also suggest that MIWI2 and MIWI2-associated piRNAs have functions beyond TE suppression. Cell Death and Differentiation (2014) 21, 783-796; doi:10.1038/cdd.2014.5; published online 24 January 2014

The Argonaute family of proteins contains two subclasses, argonaute (AGO) and p-element induced wimpy testis (PIWI), both of which are highly conserved from plants to animals. ${ }^{1}$ The AGO subfamily members (for example, AGO1-4) are expressed ubiquitously in a wide range of tissues/cell types and function through associations with miRNAs and siRNAs. ${ }^{2}$ The PIWI subfamily of proteins were initially discovered for their involvement in germline stem cell self-renewal in Drosophila, ${ }^{3}$ and later were found to repress retrotransposons in mammalian testes by interacting with PIWI-interacting RNAs (piRNAs). ${ }^{4-7}$ piRNAs represent a distinct small noncoding RNA (sncRNA) species with an average length of 25-31nt, and piRNA biogenesis is independent of the RNase III enzymes DROSHA and DICER, which are essential for the production of miRNAs and siRNAs. ${ }^{8-10}$ piRNAs can generally be divided into two groups based on their genomic origin: repeat-associated and non-repeat-associated. ${ }^{4,7,11}$ Repeatassociated piRNAs are mostly derived from retrotransposon loci and appear to be synthesized through two successive steps: primary piRNA processing and secondary piRNA cleavage achieved by the so-called 'ping-pong' amplification cycle. These occur in both the Drosophila germline and the mammalian fetal testes. ${ }^{6,11-13}$ In this model, long singlestranded piRNA precursors are transcribed from retrotransposon loci or repetitive sequences. Precursor piRNAs are further cleaved into primary, sense-orientated piRNAs, a step in which MITOPLD and MOV10L1 have recently been shown to be essential. ${ }^{14-18}$ Subsequently, MILI intrinsic endonuclease activity recognizes and cleaves secondary piRNAs, based on the feature of 10 nucleotides complementary to the primary piRNAs at the $5^{\prime}$ end. ${ }^{19,20}$ In this way, repetitive sequences are processed into mature piRNAs, which are then incorporated into their effector complexes that can then enter the nucleus and induce chromatin remodeling, or DNA methylation, to silence the repetitive sequences from which they are derived. ${ }^{11,21}$ In this sense, the PIWI-piRNA pathway is believed to be an RNA-based genome defense system, which can degrade and inactivate retrotransposons at both the transcriptional and posttranscriptional levels in host cells. ${ }^{13,21-25}$ However, the existence of only primary piRNAs, but not secondary piRNAs, in Drosophila ovarian somatic cells, and in pachytene spermatocytes and round spermatids in the murine testes, suggests that it may have other novel functions beyond TE suppression. ${ }^{18,26,27}$

In mice, three PIWI family proteins have been identified, including MIWI (PIWIL1), MILI (PIWIL2), MIWI2 (PIWIL4), all of which are exclusively expressed in the testis. ${ }^{28-30}$ MILI expression starts in primordial germ cells (PGCs) at

\footnotetext{
${ }^{1}$ Department of Physiology and Cell Biology, University of Nevada Reno School of Medicine, 1664 North Virginia Street, Mailstop 0575, Reno, NV 89557, USA and ${ }^{2}$ Washington State University, Abelson Hall Room 507, Pullman, WA 99164, USA

${ }^{*}$ Corresponding author: W Yan, Department of Physiology and Cell Biology, University of Nevada School of Medicine, Center for Molecular Medicine, Room 207B, 1664 North Virginia Street, MS 0575, Reno, NV 89557, USA. Tel: +775 7847765 (Office) +775 7844688 (Lab); Fax: +775 784 4362; E-mail: wyan@ medicine.nevada.edu Keywords: MIWI2; PIWI; piRNAs; conditional knockout; meiosis; transposon

Abbreviations: TE, transposable element; LINE, long interspersed element; IAP, intracisternal A-particle; piRNAs, Piwi-interacting RNAs; AGO, argonaute; PIWI, p-element induced wimpy testis; sncRNAs, small non-coding RNAs; miRNA, microRNA; siRNA, small interfering RNA; PGC, primordial germ cell; NMD, nonsense mediated decay; cKO, conditional knockout; DSB, double strand break; HE, hematoxylin and eosin staining; FACS, fluorescence activated cell sorting

Received 16.10.13; revised 21.12.13; accepted 30.12.13; Edited by JC Marine; published online 24.1.14
} 
embryonic day 12.5 (E12.5) and lasts until the round spermatid stage in adult testes, whereas MIWI2 is expressed only in PGCs in fetal testes and MIWI is exclusively present in the pachytene spermatocytes and round spermatids in postnatal testes. ${ }^{11,28,29}$ The differential expression and localization patterns suggest that the three PIWI proteins may have differential functions during testicular development and spermatogenesis. Indeed, global inactivation of each of the three PIWI proteins leads to differential phenotypes, which is consistent with the notion that the three have non-redundant functions despite the high degree of conservation in their domain structures. ${ }^{22,27,31}$ Mili or Miwi2 global knockout male mice exhibit meiotic prophase I defects that are correlated with desuppression of LINEI and IAP retrotransposons, ${ }^{20,32}$ whereas Miwi knockouts display a spermiogenic arrest in step-4 round spermatids, which is also associated with increased postmeiotic LINE1 activities. ${ }^{9,29}$ Recently, Mili was conditionally inactivated in spermatogenic cells in postnatal testes and this study suggests that MILI and the piRNA pathway are required to posttranscriptionally silence L1 in pachytene spermatocytes even in the presence of normal L1 DNA methylation. ${ }^{33}$ In fetal gonads, MILI partners mainly with sense piRNAs derived from the cleaved transposon transcripts, whereas MIWI2 associates with the majority of antisense piRNAs believed to act as guides for TE degradation or DNA methylation-mediated TE silencing during PGC reprograming between E13.5 and E15.5. ${ }^{21,24,30}$ In adult mice testes, MIWI binds a wealth of primary/sense piRNA transcripts termed pachytene piRNAs, majority of which are derived from 214 large genomic piRNA clusters depleted of repetitive sequences, ${ }^{34}$ indicative of the TEindependent function of pachytene piRNAs. ${ }^{9,20,35}$

Miwi2 knockout studies have clearly demonstrated an essential role of $\mathrm{MIWI} 2$ in suppressing retrotransposons during PGC development in the fetal testes. ${ }^{30}$ However, the early meiotic arrest phenotype precludes further analyses of a potential role of MIWI2 in late meiotic or haploid phase of spermatogenesis. Moreover, failure of the MIWI2-piRNA pathway to induce remethylation of TE loci after global demethylation occurs in PGCs between E13.5 and birth in mice. ${ }^{30,36,37}$ However, germ cell death and depletion does not happen until postnatal day 12, when PGCs have developed into late zygotene and early pachytene spermatocytes. TE activation, in theory, is supposed to cause transposition of TEs, which usually lead to excessive DNA double-strand breaks (DSBs) and thus cell death. ${ }^{38}$ However, Miwi2-null PGCs and prospermatogonia apparently not only survive TE activation, but even manage to finish the mitotic phase (that is, spermatogonial proliferation and differentiation) and enter the meiotic phase, before finally arrested in prophase I at late zygotene stage during the first wave of spermatogenesis. This raises many questions, for example, does persistent TE activation cause active transposition? If so, is active transposition compatible with mitosis of PGCs/prospermatogonia? Is the zygotene arrest phenotype in Miwi2-null testes caused by TE activation? What is the consequence of persistent TE activation? To answer these critical questions, we generated a Miwi2 loxp mouse line and conditionally inactivated Miwi2 in male germ cells at various developmental stages. Our data demonstrate that an essential role of MIWI2 is confined to
PGCs during fetal testicular development and Miwi2 is dispensable for Sertoli cell or postnatal male germ-cell development. Furthermore, Miwi2 deficiency causes multiple defects including TE desuppression, TE-induced DNA DSBs, aberrant histone modifications and genome-wide alteration of mRNA transcriptome, which may all contribute to the early meiotic arrest phenotype observed in Miwi2 global or conditional knockouts.

\section{Results}

Generation of global and germ cell-specific Miwi2 knockout mice. MIWI2 protein consists of two well-conserved domains (Figure 1a) one is the PAZ domain essential for small RNA recognition; the other is the PIWI domain associated with the slicer activity found in members of the Argonaute family at the C-terminus. ${ }^{4,28,30}$ We engineered a targeting construct (Figure 1a) in which two loxp cassette sequences were inserted into introns 8 and 12, respectively. Through homologous recombination, we successfully generated a Miwi2 loxp allele (Miwi2 ${ }^{\text {lox }}$ ) with two loxp sequences flanking exons 9 and 12 in mouse embryonic stem cells, as confirmed by Southern blot analyses (Figure 1b). Deletion of exons 9-12 by Cre-mediated recombination would cause frame shift, leading to nonsense mRNA decay (NMD) pathway-mediated degradation of the mutant Miwi2 transcripts. PCR-based genotyping could readily distinguish the loxp allele from the WT allele (Figure 1c) and genotypes of conditional knockout mice could be determined in conjunction with PCR-based genotyping on the transgenic Cre allele (Figure 1c).

The homozygous Miwi2 loxp mice were indistinguishable from WT mice, suggesting the insertion of the loxp sequences into the introns did not compromise Miwi2 functions. To prove that deletion of Miwi2 loxp allele leads to a null allele, we crossed the Miwi2 loxp line with the Ella-Cre line, which expresses Cre in all cells during an early embryonic development and thus, can be used to generate global KO mice. Ella-Cre; Miwi2 ${ }^{\text {lox/4 }}$ mice phenocopied Miwi2 global KO mice ${ }^{30}$ (see below for details), suggesting that deletion of the floxed exons 2-9 indeed generated a Miwi2-null allele.

We also examined MIWI2 protein expression in WT fetal testes between E12.5 and P5, and in the testes of Ella-Cre; Miwi2 $^{\text {lox/4 }}$ mice at E13.5 (Supplementary Figure S1). Consistent with previous reports, ${ }^{12,30}$ MIWI2 was abundantly expressed in PGCs in E13.5 testes, and in prospermatogonia in E15.5, E18.5 and newborn (P0) testes. In Miwi2-null testes at $E 13.5$, no signals were detected, suggesting that we indeed generated a true null Miwi2 allele after CRE-mediated recombination.

The early meiotic arrest in Miwi2 global KO mice precludes further analyses of the potential roles of MIWI2 in late meiotic and haploid phases of spermatogenesis. To further explore potential roles of MIWI2 in the meiotic and haploid phases of spermatogenesis, we crossed Miwi2 loxp mice with three germ cell-specific Cre lines to inactivate Miwi2 in the male germ line at different developmental time points. The $D d x 4-C r e$ line has been used to delete a floxed allele in PGCs/prospermatogonia with an onset of CRE expression 
a
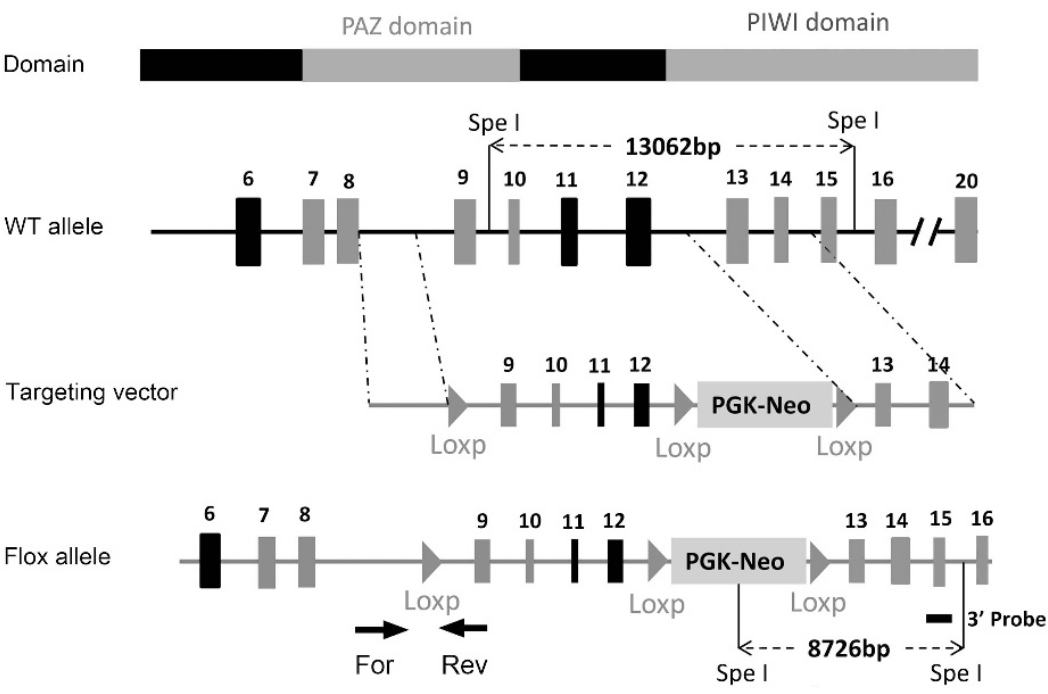

b

c
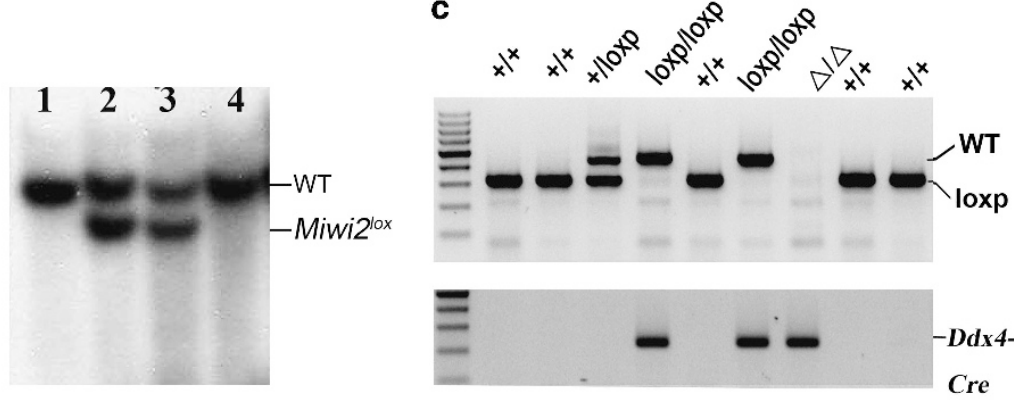

d

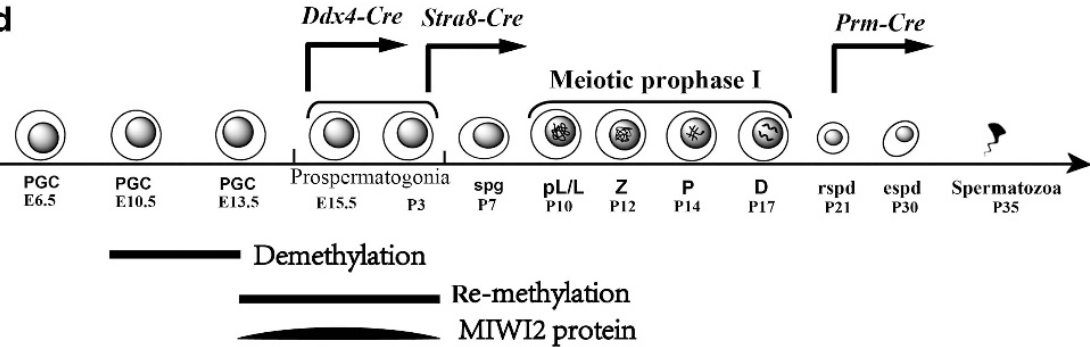

Figure 1 Generation of Miwi2 conditional knockout mice. (a) Strategy for generating a floxed Miwi2 allele in murine embryonic stem (ES) cells. In the targeted ES cells, exons 9-12 are flanked by two loxp sequences, and Cre-mediated recombination leads to the deletion of the four exons, which causes a frame shift and a complete lack of MIWI2 protein production. Arrows depict the locations of PCR genotyping primers (For, forward primer; Rev, reverse primer). The restriction enzyme Spel was used to digest genomic DNA in Southern blot analyses shown in panel b. (b) A representative Southern blot showing two WT ES cell clones (Lanes 1 and 4) and two targeted ES cell clones (Lanes 2 and 3) carrying one WT allele (13062 bp) and the other Miwi2 floxed allele (Miwi2 ${ }^{\text {lox }}$ ( 8726 bp). (c) Representative PCR genotyping results showing tail samples with different genotypes (WT $=438 \mathrm{bp}$, miwi2 ${ }^{10 x}=315 \mathrm{bp} ; \mathrm{Ddx} 4-\mathrm{Cre}=240 \mathrm{bp}$ ). (d) A schematic diagram showing the timeline of male germ-line development and the onset of Cre expression in three germline-specific Cre deletor lines

at $\mathrm{E} 15.5,{ }^{39}$ whereas Stra8-Cre mice display CRE expression in prospermatogonia at P3 although the full penetrance of CRE activity is not reached until pachytene spermatocytes at $\mathrm{P} 12^{10,40,41}$ (Figure 1d). The Prm-Cre line has Cre expression starting in round spermatids, but the protein expression may be delayed due to posttranscriptional regulation ${ }^{42}$ (Figure 1d). By crossing the three Cre deletor lines with Miwi2 loxp mice, we generated three male germ cell-specific Miwi2 cKO lines, in which Miwi2 is inactivated in prospermatogonia (Ddx4-Cre; Miwi2 $^{\text {lox/4 }}$ ), pachytene spermatocytes (Stra8-Cre; Miwi2 ${ }^{\text {lox/4 }}$ ) and spermatids (Prm-Cre; Miwi2 ${ }^{\text {lox/4}}$ ) (Figure 1d and Supplementary Figure S2).
MIWI2 is dispensable for postnatal testicular germ-cell development. Progeny of all four cKO lines, including heterozygous (Cre; Miwi2 ${ }^{+/ l o x}$ ) and homozygous (Cre; Miwi $\left.2^{\text {lox/4}}\right)$, all developed and behaved normally, and were grossly indistinguishable. However, fertility tests indicated that Ddx4-Cre; Miwi2 ${ }^{\text {lox/4 }}$ and Ella-Cre; Miwi2 ${ }^{\text {lox/4 }}$ male mice were infertile, whereas Stra8-Cre; Miwi2 ${ }^{\text {lox/4 }}$ and Prm-Cre; Miwi $2^{\text {lox/4 }}$ male mice were as fertile as WT males. Upon examination of the testes, we found that testes from Ddx4-Cre; Miwi2 ${ }^{\text {lox/4 }}$ or Ella-Cre; Miwi2 ${ }^{\text {ox/4 }}$ mice were much smaller than age-matched testes from Stra8-Cre; Miwi2 ${ }^{\text {lox/4 }}$ or Prm-Cre; Miwi2 ${ }^{\text {lox/A }}$ mice (Figures $2 \mathrm{a}$ and b). Testis weight 
a
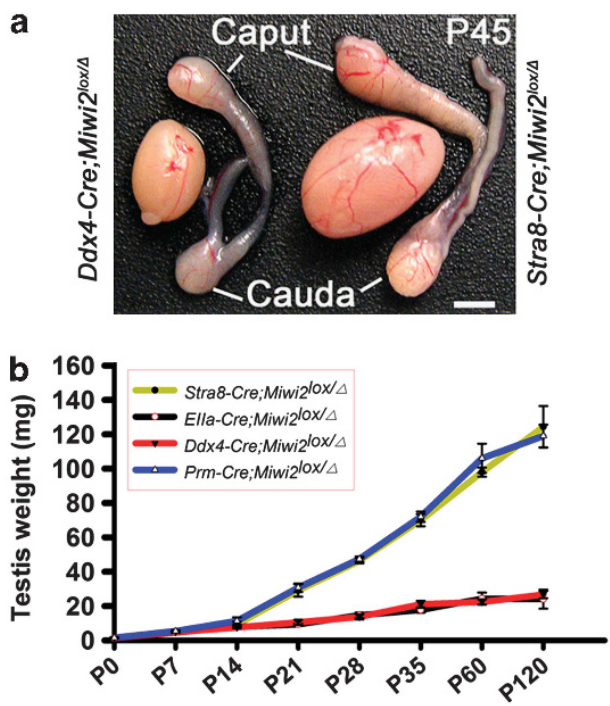
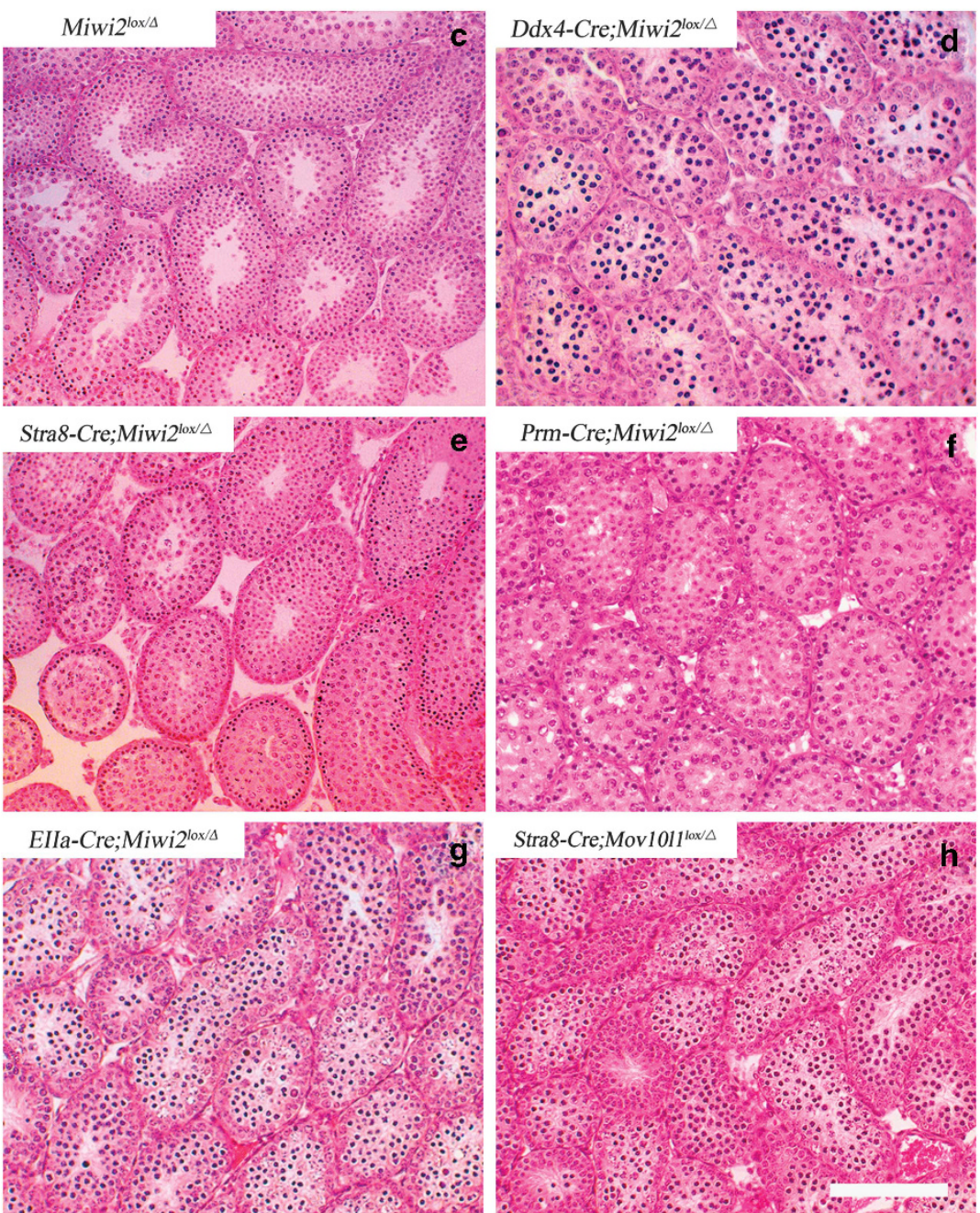

Figure 2 Miwi2 is required between embryonic day 15.5 (E15.5) and postnatal day 3 (P3), but dispensable after P3 during murine testicular development. (a) A representative image showing the testes of Ddx4-cre; Miwi2 ${ }^{\text {lox/ }}$ male mice were smaller than Stra8-Cre; Miwi2 ${ }^{\text {lox } / 4}$ testes, which were similar to WT controls. (b) Testis growth curves in male mice with different genotypes indicated. Stra8-Cre; Miwi2 ${ }^{\text {lox/4 }}$ and Prm-Cre; Miwi2 ${ }^{\text {lox } / \Delta}$ WT male mice displayed the same testicular growth rate as WT controls, which are omitted here. (c-h) Testicular histology of two control (Miwi2 ${ }^{+/ 10 x}$ as a negative control, whereas Stra8-Cre; Mov10/1 $1^{\text {lox/4 }}$ as a positive control), and four male germ cell-specific Miwi2 conditional knockout mouse lines. Note that all testes samples were from postnatal day 21 (P21). Scale bar $=200 \mu \mathrm{m}$ 
of Stra8-Cre; Miwi2 $2^{\text {lox/4 }}$ or Prm-Cre; Miwi2 ${ }^{\text {lox/4 }}$ male mice increased (Figure 2b) at similar rates to those of WT or heterozygous littermates during postnasal testicular development (data not shown). However, the testes of Ddx4-Cre; Miwi2 $^{\text {lox/4 }}$ or Ella-Cre; Miwi2 ${ }^{\text {lox/4 }}$ male mice stopped gaining weight after $\mathrm{P} 7$, and the average testis weight was only $\sim 16 \%$ of that of Stra8-Cre; Miwi2 ${ }^{\text {lox/4 }}$ or Prm-Cre; Miwi2 ${ }^{\text {lox/4 }}$ males at the age of 2 months (Figure $2 b$ ). The cessation of testis growth suggests severe spermatogenic disruptions in Ddx4-Cre; Miwi2 ${ }^{\text {lox/4 }}$ (hereafter called Miwi2 cKO) and EllaCre; Miwi2 ${ }^{\text {lox/4 }}$ (hereafter called Miwi2 uKO) mice. Histological examination of paraffin-embedded, Hematoxylin-Eosin $(\mathrm{HE})$-stained testis sections revealed that at P21, only zygotene-like spermatocytes were present as the most advanced mature male germ cell type in Miwi2 cKO and Miwi2 uKO mice testes (Figures $2 \mathrm{~d}$ and $\mathrm{g}$ ). This was in contrast to the first wave of normal spermatogenesis observed in Miwi2 ${ }^{+/ l o x}$, Stra8-Cre; Miwi2 ${ }^{\text {lox/4 }}$ or Prm-Cre; Miwi2lox/4 testes, characterized by the presence of round spermatids in the luminal compartment of the seminiferous epithelium (Figures 2c, e and f). These data demonstrate that spermatogenesis was arrested at meiotic prophase I in the testes of Miwi2 cKO and Miwi2 uKO mice, but was normal in Miwi2 $+/ l o x$, Stra8-Cre; Miwi2 ${ }^{\text {lox/4}}$, or Prm-Cre; Miwi2 ${ }^{\text {lox/4 }}$ testes. Interestingly, the testicular histology of Miwi2 cKO and Miwi2 uKO mice was very similar to that of Stra8-Cre; Mov1011 $10 x / 4$ mice ${ }^{11}$ and global Mov10l1 KO mice, ${ }^{16,18}$ both of which display an early meiotic arrest (Figure $2 \mathrm{~h}$ ). Collectively, these data imply that Miwi2 has a temporally restricted role, that is, Miwi2 is required only for PGC/ prospermatogonial development, but is dispensable for postnatal male germ-cell development.

Meiotic arrest during testicular development in Miwi2 cKO mice. We next analyzed the histology of developing testes to determine developmental defects in Miwi2 cKO testes. Similar histology was observed in both Miwi2 cKO and control (Miwi2 ${ }^{+/ l o x}$ ) testes from P4 to P7 (Figure 3a). However, at P10, there appeared to be a delay in male germcell development because the control testes contained numerous leptotene spermatocytes, whereas only few or no leptotene spermatocytes were present in Miwi2 cKO testes (Figure 3a). To confirm this, we examined $\gamma \mathrm{H} 2 \mathrm{Ax}$ expression because $\gamma \mathrm{H} 2 \mathrm{Ax}$ is a specific marker for DNA DSBs and thus, is highly expressed in the nuclei of meiotic male germ cells from leptotene to pachytene spermatocytes. ${ }^{43}{ } \mathrm{H} 2 \mathrm{Ax}$ immunofluorescent staining assays revealed that Miwi2 cKO testes contained much fewer leptotene spermatocytes than control testes at P10 (Figure 3c), supporting that, indeed, germ cell development is delayed in Miwi2 cKO testes. At P14 and thereafter, a meiotic block became apparent in Miwi2 cKO testes, based on that only zygotene-like spermatocytes were present as the most advanced germ cell types, whereas pachytene spermatocytes already existed in control testes at P14 (Figure 3a). Immunofluorescent staining of SCP3 (a meiotically specific component of the synaptonemal complex structure) and $\gamma \mathrm{H} 2 \mathrm{Ax}$ of chromatin spreads were prepared using single cells from P21 seminiferous tubules of Miwi2 cKO and control testes revealed a complete lack of pachytene spermatocytes in Miwi2 cKO testes, whereas abundant leptotene, zygotene and pachytene spermatocytes were present in control testes (Supplementary Figure S3), further confirming a meiotic arrest at the zygotene stage of meiotic prophase I in Miwi2 cKO testes. The histology of the cKO testes at the age of 10 months showed seminiferous tubules containing few germ cells up to the zygotene stage or a complete lack of germ cells, resembling the 'Sertoli-only syndrome' in humans (Figure 3d). To further confirm that P10 is the onset of histologically discernable spermatogenic disruptions, we immunohistochemically labeled all germ cells using the GCNA antibody ${ }^{44}$ (Figure 3b). By counting GCNA-positive cells (that is, male germ cells) per cross section (in round or close to round shape) of the seminiferous tubule, a significant decrease in germ cell number within the seminiferous tubules was first observed between $\mathrm{P} 10$ and $\mathrm{P} 14$ and the reduced number of male germ cell within the seminiferous tubules appeared to persist thereafter (Figure 3e). Taken together, inactivation of Miwi2 in PGCs/prospermatogonia at E15.5 leads to a delayed meiotic progression, and ultimately a meiotic arrest at the zygotene stage of meiosis prophase I during postnatal testicular development.

TE activation and increased DNA DSBs in Miwi2-null male germ cells. Previous reports have shown that global inactivation of Miwi2 leads to a failure of demethylated TEs to be remethylated in PGCs between E13.5 and E15.5. ${ }^{11,24}$ To understand the extent and consequences of TE derepression, we analyzed mRNA levels of various TEs and also protein levels of LINE1 and IAP, as well as $\gamma \mathrm{H} 2 \mathrm{Ax}$ levels for DSBs (Figures $4 a$ and b). Among five types of TEs analyzed, levels of LINE1 and IAP mRNAs were significantly upregulated in Miwi2 cKO testes compared with WT testes at P12 (Figure 4a). Consistently, both ORF1 of LINE1 and IAP proteins were abundantly expressed in Miwi2 cKO testes at P12 (Figure 4b), and the levels were comparable with those in either Mov1011 global $^{45}$ or cKO testes at P12 (Figure 4b). In contrast, levels of ORF1 and IAP were barely detectable in control $\left(\right.$ Miwi2 $\left.{ }^{+/ 10 x}\right)$ testes at the same age. Previous reports have shown that both LINE1 and IAPS are derepressed due to a failure in remethylation of these TE loci in PGCs, leading to a persistent TE activation from E15.5 onward. ${ }^{16,44}$ Indeed, LINE1/ORF1 protein was detected abundantly in both newborn and P7 testes, whereas control testes at the same ages were largely negative in LINE1/ORF1 staining (Figure 4c). To determine whether persistent activation of LINE1 and IAPS could have caused transposition and DSBs, we examined $\gamma \mathrm{H} 2 \mathrm{Ax}$ expression in newborn and P7 testes of control and Miwi2 cKO mice (Figure 4c). Interestingly, while there were hardly any $\gamma \mathrm{H} 2 \mathrm{Ax}$-positive cells in control newborn and $\mathrm{P} 7$ testes, many male germ cells were positive for $\gamma \mathrm{H} 2 \mathrm{Ax}$ staining in the newborn or P7 Miwi2 cKO testes (Figure 4c) and there appeared to be more $\gamma \mathrm{H} 2 \mathrm{Ax}$-positive cells in $\mathrm{P} 7$ than in newborn Miwi2 cKO testes, suggesting an increasing incidence of DSBs in Miwi2-null prospermatogonia or spermatogonia. However, the number of $\gamma \mathrm{H} 2 \mathrm{Ax}$-positive germ cells appeared to be much less than that of ORF1- or IAP-positive cells. The discrepancy may reflect the fact that persistent TE activation can cause either no or efficient transpositional events, leaving fewer cells with DSBs. 
a
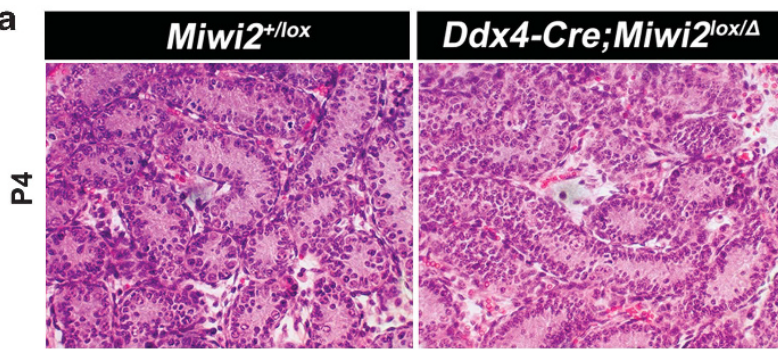

ล

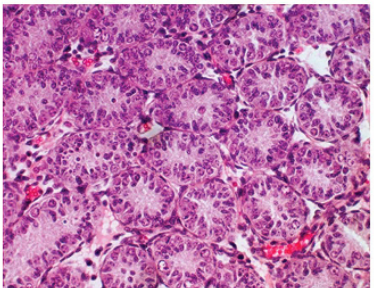

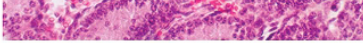
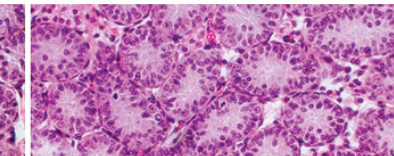

201/20
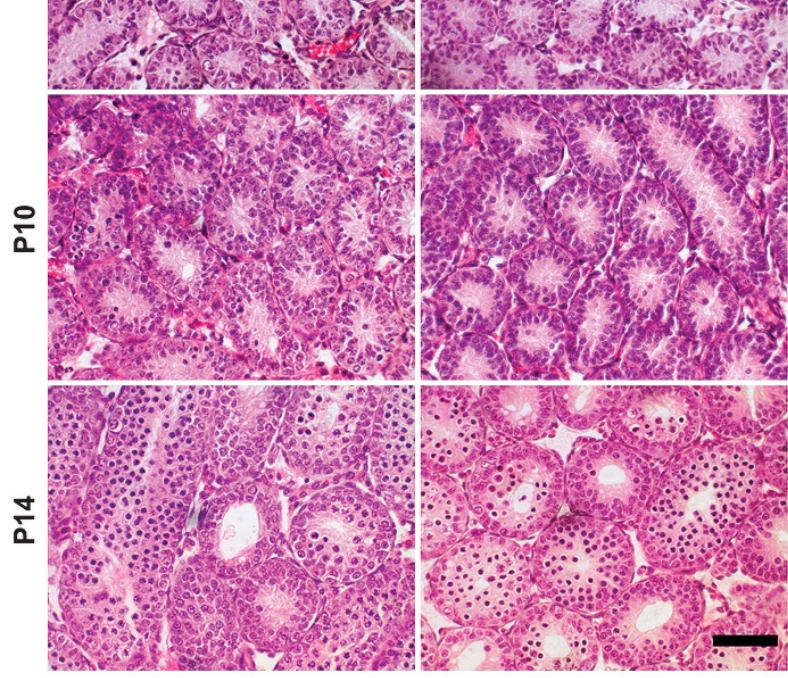

C
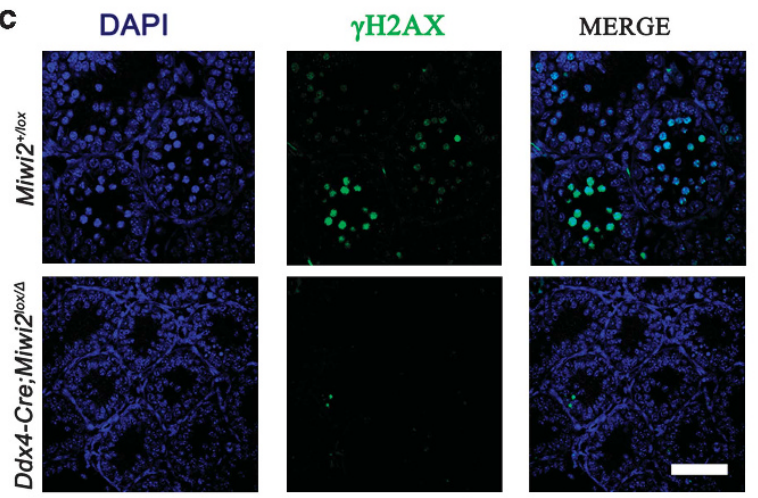

d

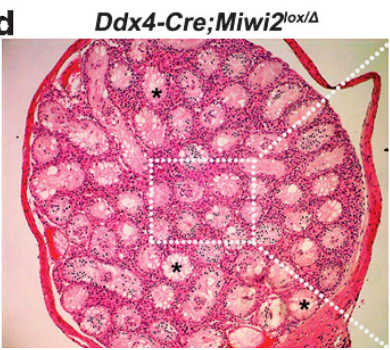

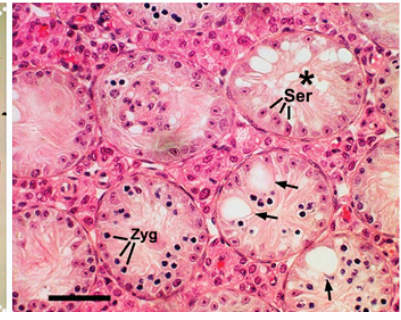

b

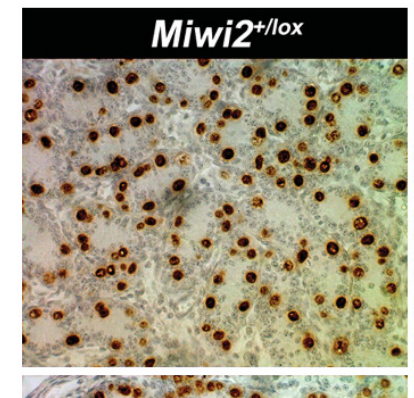

Ddx4-Cre;Miwi2'ox/4

à

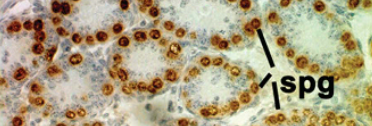

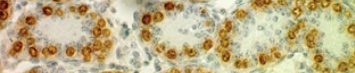

6. 35 .

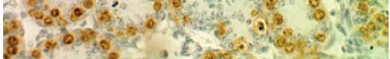

\%

웅

iิ
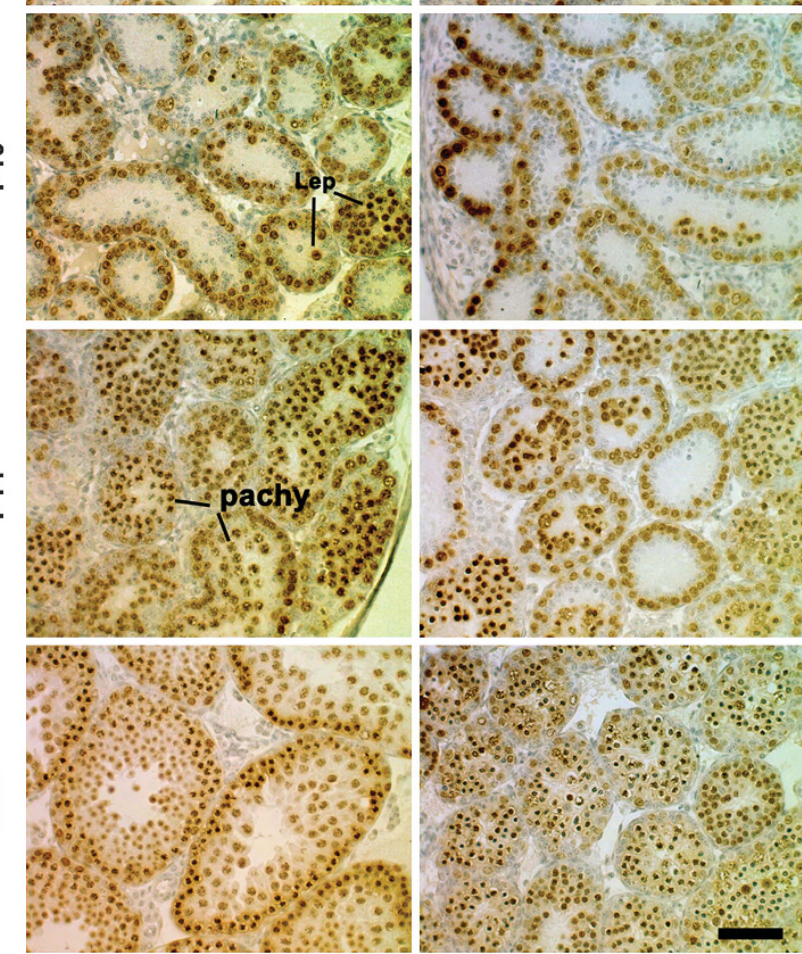

e

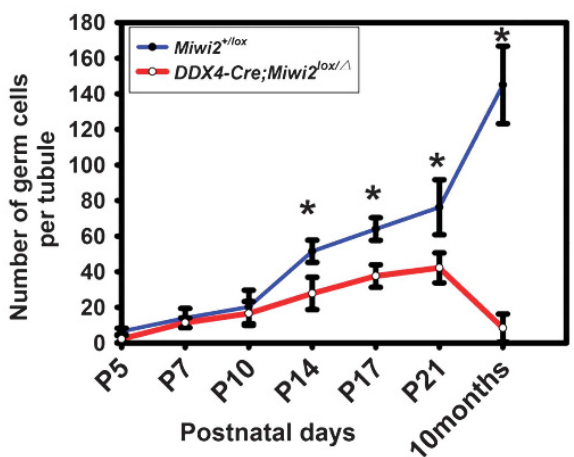


Nevertheless, persistent TE activation indeed occurred in Miwi2 cKO testes, which appeared to have caused enhanced DSBs between the reentry into mitosis (P3) and the onset of meiosis (P12).

Altered chromatin histone modification patterns in Miwi2-null male germ cells. Inactivation of Miwi2 has been shown to lead to derepression of TEs due to a failure in remethylation of TE loci during PGC reprograming. ${ }^{11,46}$ Aberrant methylation of at least one imprinted gene in Miwi2-null PGCs ${ }^{46}$ suggests that the DNA methylation defects are widespread. However, other epigenetic abnormalities in Miwi2-null PGCs and subsequent male germ-cell types have not been explored. To investigate whether MIWI2 ablation affects histone modifications, we performed immunofluorescent staining on meiotic nuclear spreads to detect trimethylation of histone $\mathrm{H} 3$ at lysine 4 (H3K4me3, an activating histone mark). We also examined trimethylation of histone $\mathrm{H} 3$ at lysine $9(\mathrm{H} 3 \mathrm{~K} 9 \mathrm{me} 3)$ and trimethylation of histone $\mathrm{H} 3$ at lysine 27 (H3K27me3), both of which are repressive histone markers. H3K4me3 modification is essential for murine male germline development because disruption of Meisetz (a meiotic methyltransferase containing a PR/SET domain and zinc-finger motif), which catalyzes the trimethylation of lysine 4 of histone 3 , causes sterility in both male and female mice. ${ }^{47}$ In Miwi2 cKO testes, the intensity of H3K4me3 immunofluorescent signals appeared to be decreased in leptotene and zygotene spermatocytes compared with the same cell types in control testes (Figure 5). Similarly, H3K9me3 staining, which usually exhibits multiple spots-like staining patterns in control spermatocytes, ${ }^{48}$ was also altered in both spermatogonia and spermatocytes in Miwi2 cKO testes, which was characterized by much smaller spots lacking smooth and sharp boundaries (Supplementary Figure S4). More strikingly, H3K27me3 immunofluorescent staining was barely detectable in spermatogonia, leptotene and zygotene spermatocytes from Miwi2 cKO testes at P21, whereas this histone mark showed strong and distinct staining patterns in the same germ-cell types in P21 control testes (Figure 6). Together, these data suggest that in addition to persistent TE activation and DSBs, Miwi2-null germ cells also have a defect in histone modifications although whether this defect is a primary effect of Miwi2 inactivation or a secondary effect due to TE derepression remains unknown.

Altered mRNA transcriptome in Miwi2-null meiotic cells. We next examined mRNA transcriptomic changes in
Miwi2-null meiotic cells using Affymetrix microarray analyses. Meiotic cells were purified from P10 Miwi2 cKO and WT testes using fluorescence activated cell sorting (FACS) based on chromatin contents ${ }^{49}$ (Supplementary Figure S5). At P10, Miwi2 cKO testes were histologically indistinguishable from those of WT testes, which mainly contained leptotene and early zygotene spermatocytes as the meiotic cell types (Figure 2a). Microarray analyses revealed that a total of 2642 mRNAs were significantly deregulated (with 1317 upregulated and 1325 downregulated; cutoff parameters used: mean difference $>10, P<0.05$, fold change $>1.2$ ) in meiotic cells purified from Miwi2 cKO testes compared with those from WT testes (Figure 7a). Interestingly, a population of transcripts derived from the Pol fragment of IAP transposons was significantly upregulated (highlighted in red in Supplementary Table S2), further validating the microarray data. Bioinformatic analyses using DAVID functional annotation pipeline ${ }^{50}$ revealed an enrichment of GO terms on transcription-related biological processes in the deregulated genes (Figure 7b), suggesting that the normal meiotic transcriptional program was disrupted in Miwi2-null meiotic cells.

The overall changes in the mRNA transcriptome in Miwi2 cKO testes prompted us to speculate that piRNAs might directly regulate the expression of protein-coding genes. We, therefore, performed bioinformatic analyses by mapping MIWI2-associated piRNAs (from datasets with an accession number E-MTAB-730) ${ }^{19}$ to the deregulated (up or downregulated) and unregulated mRNAs (as a background control) in Miwi2 cKO testes in a reverse complimentary manner (Figure 7c). Intriguingly, MIWI2-piRNAs display a clear preference to the $3^{\prime} U T R$ s. This feature is similar to that of miRNAs, which preferentially target $3^{\prime} U T R s$ and affect mRNA stability and translational efficacy. In general, MIWI2-piRNAs appeared to have a higher probability of hitting deregulated mRNAs $(\sim 17 \%)$ than unregulated mRNAs $(\sim 12 \%)$ with a preference of targeting $3^{\prime}$ UTRs. (Supplementary Table S1). Although direct evidence of piRNAs acting on mRNAs remains lacking, our bioinformatic analyses support a possibility of piRNAs-mediated posttranscriptional regulation. Overall, disrupted mRNA transcriptome is, at least in part, responsible for the zygotene arrest phenotype in Miwi2 cKO testes.

\section{Discussion}

Confined MIWI2 expression is consistent with its temporally restricted role in PGC/prospermatogonial development. Miwi2 mRNAs have been detected in both

Figure 3 Inactivation of Miwi2 in primordial germ cells (PGCs)/prospermatogonia at E15.5 does not disrupt mitotic progression of spermatogonia, but leads to delayed entry into the meiotic phase and an eventual block in the zygotene stage of prophase I during the first wave of spermatogenesis. (a) Histology of the testes from control $\left(\right.$ Miwi2 $\left.^{+/ l o x}\right)$ and Miwi2 cKO (Ddx4-Cre; Miwi2 $\left.{ }^{\text {lox/4 }}\right)$ mice at various developmental time points (P4, P7, P10 and P14). Note that the morphology and total number of germ cells are indistinguishable between control and Miwi2 cKO testes before P10. Scale bar $=120 \mu \mathrm{m}$; (b) Immunohistochemical staining of germ cell nuclear antigen (GCNA), a marker for germ cells, in developing testes of control and Miwi2 cKO mice. Typical germ cell types were labeled at specific time points during postnatal testicular

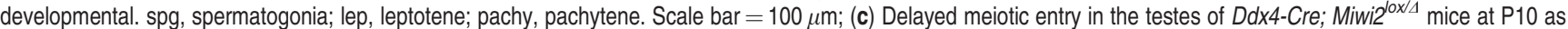
revealed by $\gamma \mathrm{H} 2 \mathrm{Ax}$ immunofluorescent staining. A large number of $\gamma \mathrm{H} 2 \mathrm{Ax}$-positive germ cells (that is, leptotene spermatocytes) are present in the control (Miwi2 ${ }^{+/ / l o x}$ ) testis, whereas only a few exist in the Ddx4-Cre; Miwi2 ${ }^{\text {lox } / \Delta}$ testis. Scale bar $=100 \mu \mathrm{m}$. (d) Testicular histology of a 10-month-old Ddx4-Cre; Miwi2 ${ }^{\text {lox/ }}$ male mouse. Numerous vacuoles can be observed in the seminiferous tubules, as indicated by arrows. Although some tubules contain only Sertoli cells (Ser), resembling 'Sertoli-only' histology in humans ( ${ }^{*}$ ), many tubules still possess meiotic germ cells, for example, zygotene spermatocytes (Zyg). (e) Quantitative analyses of germ cell number based on GCNA staining in developing testes, as shown in Panel B. Only round tubule cross sections were selected for counting germ cells and at least 20 tubules were analyzed for each time point. ${ }^{*} P<0.05$ 
a

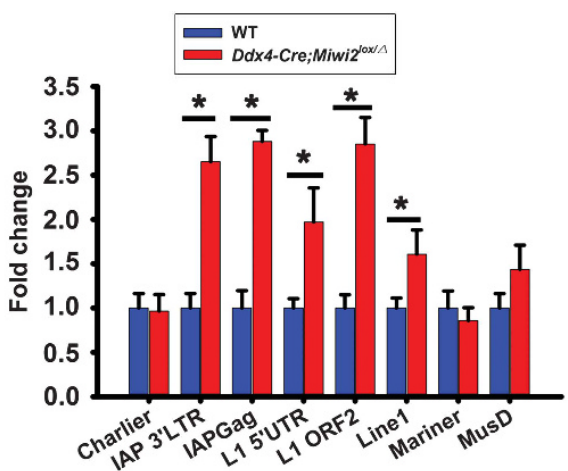

b
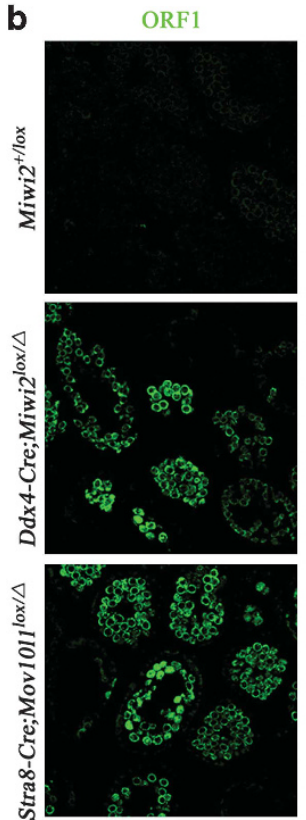

ORF $1+$ DAPI
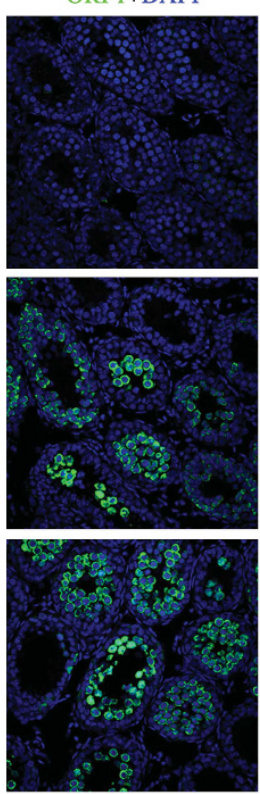

C

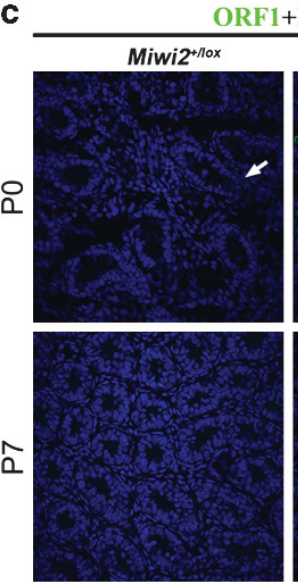

Ddx4-Cre;Miwi2/ox/4
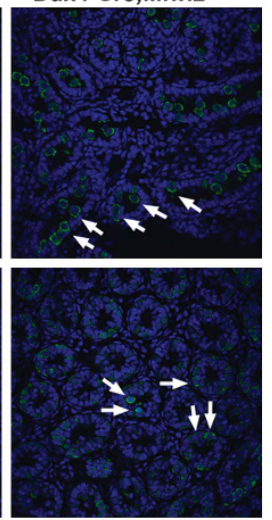

IAP
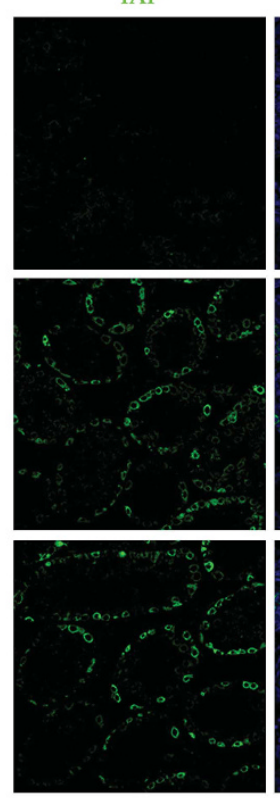

$\gamma \mathrm{H} 2 \mathrm{AX}+\mathrm{DAPI}$
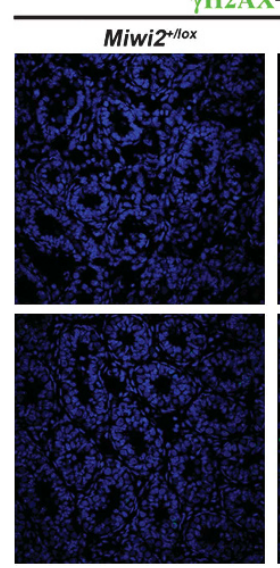

IAP+DAPI
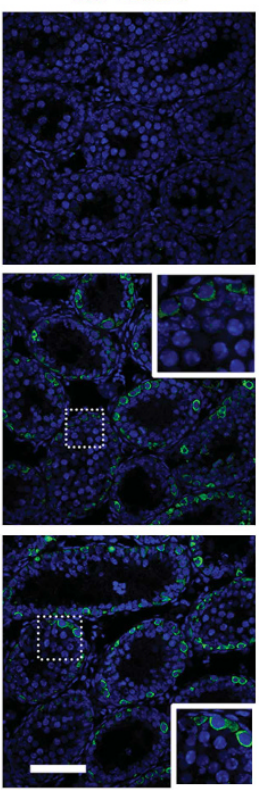

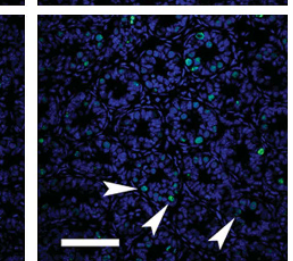

Figure 4 Activation of retrotransposons and DNA double-strand breaks (DSBs) in Ddx4-Cre; Miwi2 ${ }^{\text {lox/4 }}$ testes during postnatal development. (a) Quantitative real-time PCR (qPCR) analyses of five types of transposable elements (TEs) in WT and Ddx4-Cre; Miwi2 ${ }^{\text {lox/4 }}$ testes at P12. Transcripts of retrotransposons, such as LINE1 and IAP, were significantly upregulated in $D d x 4-C r e ;$ Miwi2 ${ }^{\text {lox/ }}$ testes, whereas the DNA transposons, for example, Charlier, Mariner and MusD, remained unaffected. ${ }^{*} P<0.05$; (b) Immunofluorescent staining of ORF1 of LINE1 and IAP in control (Miwi2 ${ }^{+/ 10 x}$ ) and Miwi2 cKO (Ddx4-Cre;Miwi2 ${ }^{\text {lox/4}}$ ) testes with Stra8-Cre; Mov1011 ${ }^{\text {lox/4 }}$ testes as a positive control. Inset represents the magnified view of the dashed area. Scale bar $=100 \mu \mathrm{m}$. (c) Immunofluorescent staining of ORF1/LINE1 and $\gamma \mathrm{H} 2 \mathrm{Ax}$ in control (Miwi2 ${ }^{+/ 10 x}$ ) and Miwi2 cKO (Ddx4-Cre; Miwi2 $\left.{ }^{\text {lox/4}}\right)$ testes at birth (P0) and P7. Although germ cells are rarely positive for either ORF1 or $\gamma \mathrm{H} 2 \mathrm{Ax}$ staining in control testes at P0 and P7, numerous prospermatogonia or spermatogonia are ORF1- (arrows) and $\gamma \mathrm{H} 2 \mathrm{Ax}$-positive (arrowheads) although $\gamma \mathrm{H} 2 \mathrm{Ax}$-positve germ cells are much fewer in Miwi2 cKO testes at the same ages. Scale bar $=100 \mu \mathrm{m}$ 

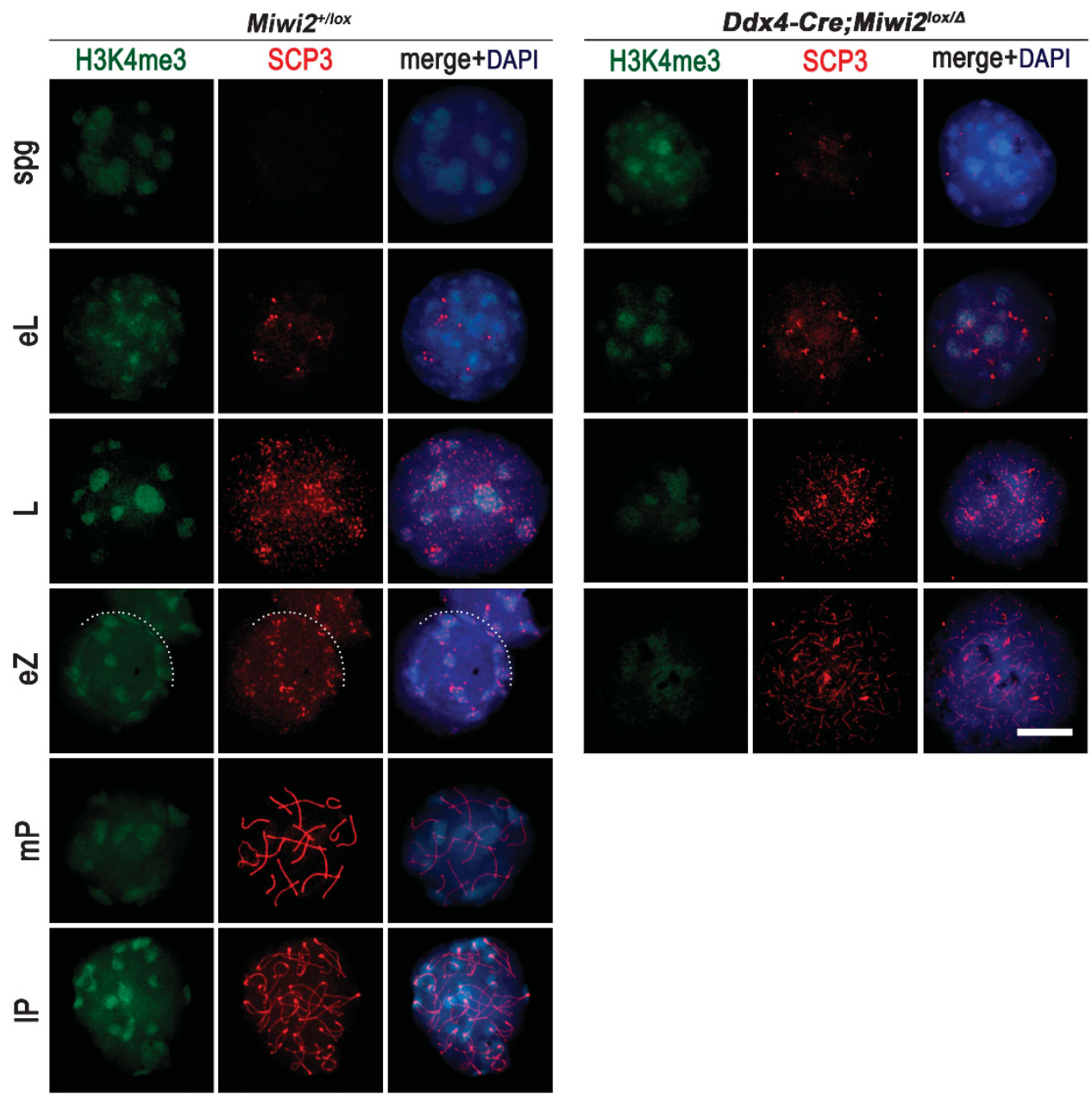

Figure 5 Immunofluorescent staining pattern of $\mathrm{H} 3 \mathrm{~K} 4 \mathrm{me} 3$ in spermatogonia and spermatocytes isolated from control (Miwi2 $\left.{ }^{+/ 10 x}\right)$ and Miwi2 $\mathrm{cKO}^{(D d x 4-C r e ;}$ Miwi2 ${ }^{\text {lox/4 })}$ testes at postnatal day 21 (P21). SCP3 staining was used to distinguish the various stages of meiotic cells. spg, spermatogonia; eL, preleptotene; L, leptotene; eZ, early zygotene; $\mathrm{mP}$, middle pachytene; IP, late pachytene. Scale bar $=5 \mu \mathrm{m}$

Sertoli cells and PGCs in fetal murine testes, ${ }^{30}$ but MIWI2 protein is mainly detected in male germ cells in fetal and neonatal murine testes (from E12.5 to P3). Using our polyclonal anti-MIWI2 antibodies, we detected MIWI2 protein expression exclusively in PGCs/prospermatogonia in fetal testes between E13.5 and E18.5, and in prospermatogonia of newborn and P2 testes. MIWI2 was undetectable in the testis after P3. The specificity of the MIWI2 antibodies used was confirmed not only by western blot analyses, in which a sole band corresponding to the correct size of MIWI2 was detected (data not shown), but also via immunofluorescent staining of the Miwi2 cKO testes at E13.5, which showed no specific signals. Our MIWI2 localization data are consistent with those previously reported, ${ }^{6,46}$ suggesting that the MIWI2 expression is strictly regulated and confined to PGCs and prospermatogonia in a narrow time window between E12.5 and $\mathrm{P} 3$. This narrow expression window implies that the role of MIWI2 is confined to PGC reprograming characterized by genome-wide demethylation followed by remethylation, which are normally completed by E13.5 and E16.5, respectively, and/or prospermatogonial development up to P3. The lack of phenotype in Stra8-Cre; Miwi2 ${ }^{\text {lox/4 }}$ and
Prm-Cre; Miwi2 ${ }^{\text {lox/4 }}$ male mice, along with the similar phenotype among Ddx4-Cre; Miwi2 ${ }^{\text {lox/4}}$, Ella-Cre; Miwi2 $2^{\text {lox/4 }}$ and Miwiw2 global KO male mice suggest that inactivation of Miwi2 in PGCs can cause a phenotype identical to that of global inactivation of Miwi2, whereas deletion of Miwi2 postnatally has no effects on spermatogenesis and other testicular functions. Our data unequivocally demonstrate that Miwi2 is required exclusively for PGC/prospermatogonia development, and Miwi2 is dispensable in postnatal testes.

Persistent TE activation is compatible with mitotic cell cycle progression of PGCs and spermatogonia. Although MIWI2 deficiency has been correlated with failure in remethylation of many TE loci and consequent TE activation, ${ }^{4,6,46}$ the underlying mechanism and consequences of TE derepression, as well as other defects associated with MIWI2 inactivation still remain unknown. By analyzing LINE1 and IAP at both mRNA and proteins levels, we demonstrate in this study that these two types of TEs are indeed activated in the absence of MIWI2 in PGCs/prospermatogonia. Most importantly, activated TEs, 

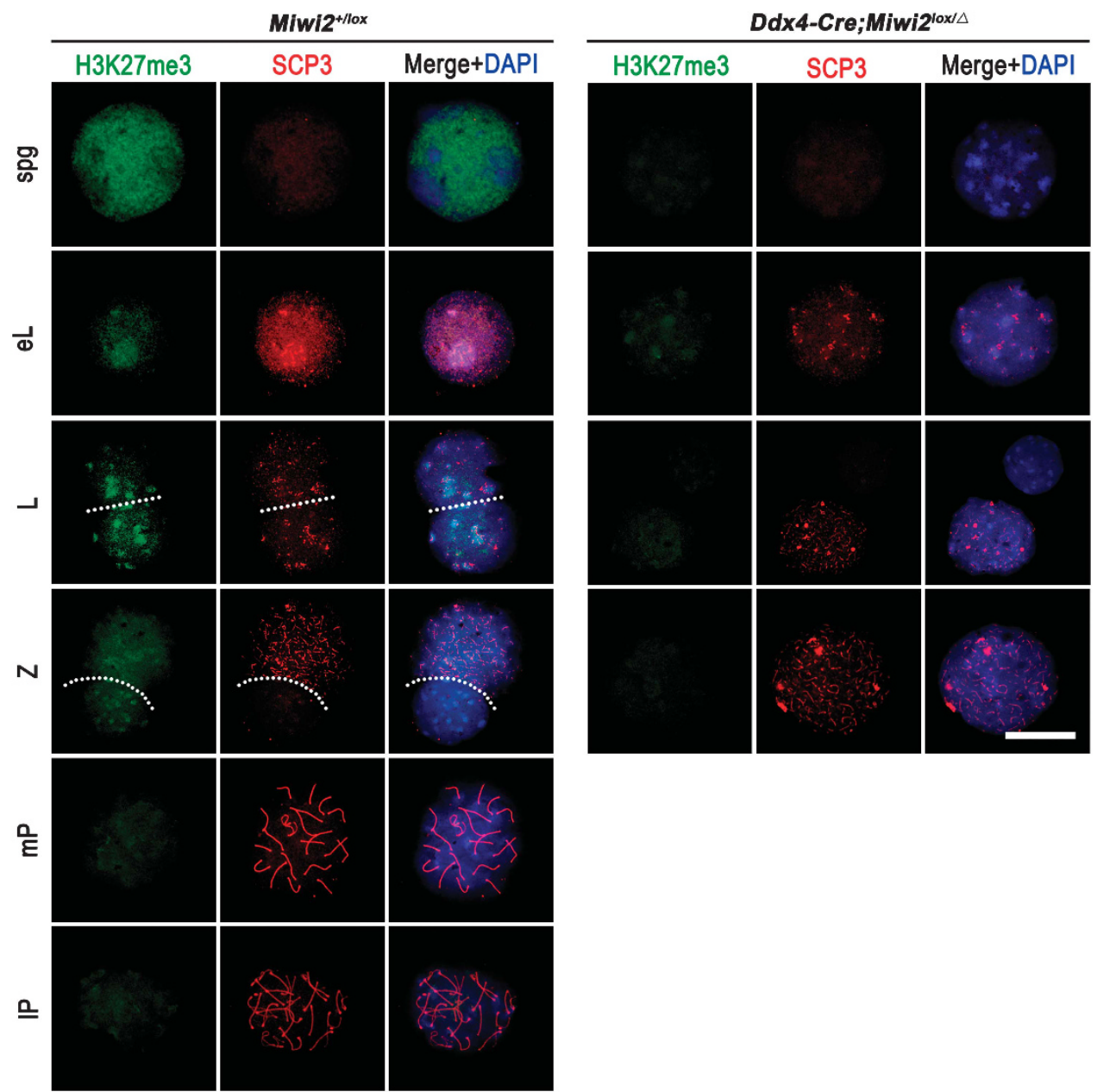

Figure 6 Immunofluorescent staining pattern of H3K27me3 in spermatogonia and spermatocytes isolated from control (Miwi2 ${ }^{+1 / 0 x}$ ) and Miwi2 cKO (Ddx4-Cre; Miwi2 ${ }^{\text {lox/4 }}$ testes at postnatal day 21 (P21). SCP3 staining was used to distinguish the various stages of meiotic cells. spg, spermatogonia; eL, preleptotene; L, leptotene; eZ, early zygotene; $\mathrm{mP}$, middle pachytene; IP, late pachytene. Scale bar $=5 \mu \mathrm{m}$

as represented by significantly enhanced expression of both LINE1 and IAP proteins, persist from PGCs/prospermatogonia all the way through to zygotene spermatocytes that are blocked and ultimately depleted from the seminiferous epithelium. This observation is in sharp contrast to a recent report showing that ORF1 is not upregulated in spermatogonia in a male germ cell-specific Mili conditional knockout line $($ Stra8-Cre; Millox/4 $){ }^{33}$ This discrepancy may result from an incomplete CRE-mediated gene deletion because the Stra8-Cre deletor line has been shown to reach full penetrance of CRE-mediated recombination in pachytene spermatocytes, although CRE expression starts in spermatogonia at postnatal day $3 .^{10}$ Interestingly, despite the abundant expression of both LINE1 and IAPs in prospermatogonia in Miwi2 cKO testes, reentry of prospermatogonia into the mitotic cell cycle and differentiation into spermatogonial stem cells and differentiating spermatogonia appear to be unaffected because no obvious difference in cellular compositions and histology was observed from newborn up to P7 between control and Miwi2 cKO testes. Persistent LINE1 and IAP activation without causing discernable disruptions during the entire mitotic phase of the first wave of spermatogenesis suggests that persistent TE activation and potentially active TE transposition are compatible with mitotic cell cycle progression of prospermatogonia and spermatogonia. Although DSBs appeared to be progressively increased in spermatogonia from P0 to P7 in Miwi2 cKO testes, the majority of spermatogonia with active LINE1 and IAP protein expression were $\gamma \mathrm{H} 2 \mathrm{Ax}$-negative, suggesting those cells, despite potentially active ongoing transpositional events, do not have DSBs, which may explain why there is no discernable cell depletion in Miwi2-null spermatogonia in early postnatal testes up to P7. Therefore, active TE activation does not necessarily causes enhanced DSBs sufficient to trigger the mitotic cell cycle-checkpoint mechanism leading to cell death. Interestingly, when type B spermatogonia entered meiosis and became preleptotene and then leptotene spermatocytes, an obvious delay in meiotic cell cycle progression was noticed at P10, which may represent an early sign of triggering the meiotic cell cyclecheckpoint mechanism. It is highly likely that extensive and persistent TE transpositions during the entire mitotic/ spermatogonial phase may have caused some major changes in chromosomal structures. However, those TE-inflicted 

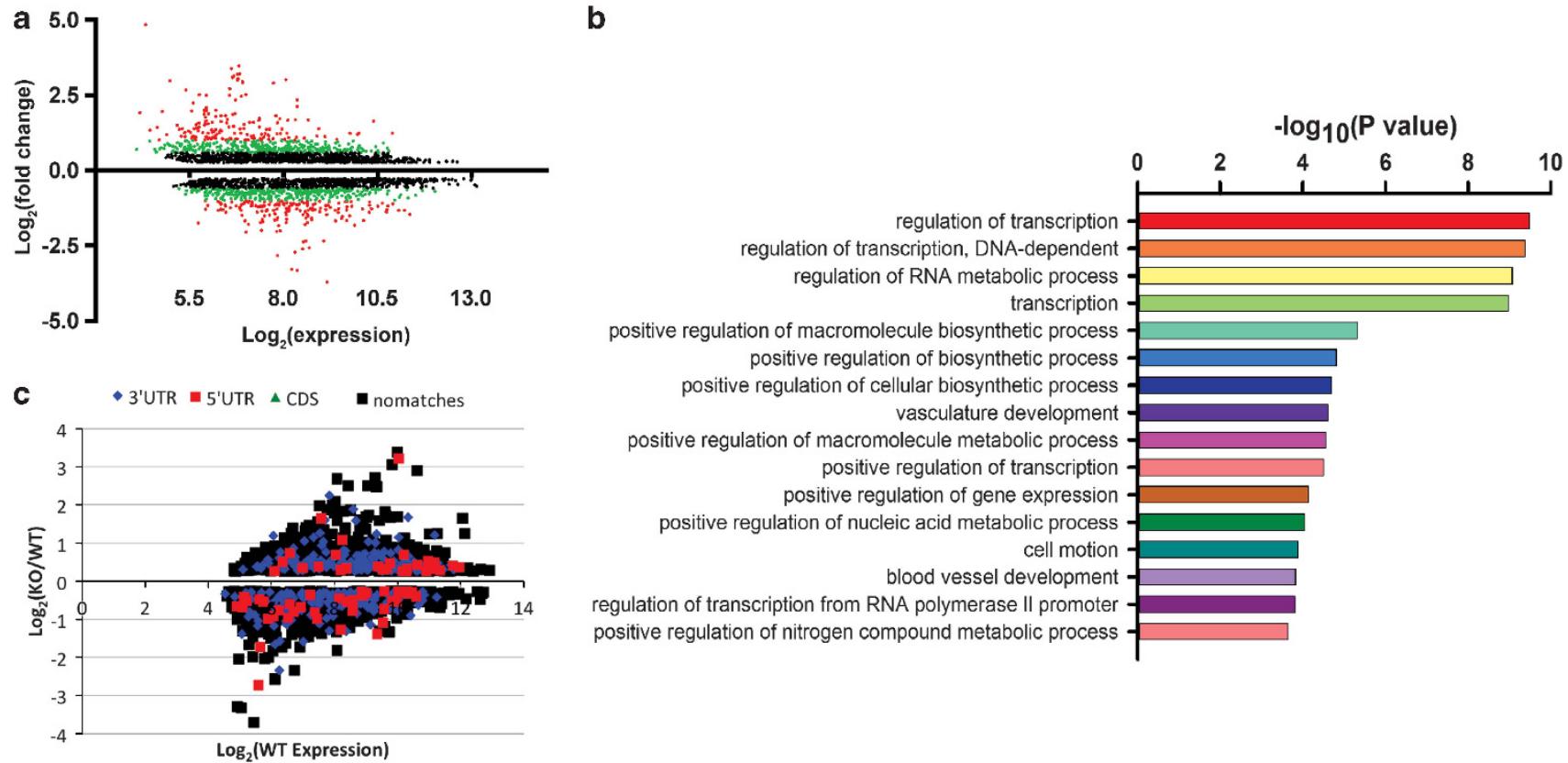

Figure 7 Microarray analyses of mRNA transcriptome in spermatocytes purified from WT and Ddx4-Cre; Miwi2 ${ }^{\text {lox } / 4}$ testes at postnatal day 11 (P11). (a) A scatter plot showing a total of 2642 up or downregulated mRNA transcripts (mean $>10, P<0.05$, fold change $(F C)>1.2$ ). Black dots: $1.2<F C<1.5 ; G r e e n$ dots: $1.5<F C<2.0 ;$ Red dots: FC > 2.0; (b) GO term analyses revealing an enrichment of genes involved in transcription-related biological processes among deregulated genes. (c) Bioinformatic analyses of potential complementary annealing between MIWI2-associated piRNAs and deregulated mRNA transcripts based on the microarray analyses

chromosome structural changes appear to be compatible with the mitotic cell cycle progression, but can be identified by the meiotic cell cycle-checkpoint mechanism in late zygotene stage of meiosis, when duplicated chromosomes start to align and pair to prepare for the upcoming homologous recombination-based crossover in the pachytene stage of meiosis. Future studies on the actual extent of TE transpositional events in Miwi2-null spermatogonia are needed to substantiate this hypothesis. Nevertheless, we can conclude based on our current data that persistent TE activation in spermatogonia due to MIWI2 deficiency is compatible with mitotic cell cycle progression of spermatogonia, but can trigger prophase I arrest in zygotene stage of meiotic cell cycle progression. This also explains why inactivation of MIWI2, which is only expressed in PGCs/ prospermatogonia from E13.5 to $\mathrm{P} 3$, does not cause a spermatogenic arrest in the mitotic phase (that is, spermatogonial development), but leads to an early meiotic prophase I arrest; and this delayed disruption reflects defects originated from the spermatogonial stage, but manifested in early meiotic phase most probably due to the incompatibility of TE activation-induced transpositional events with meiotic cell cycle progression.

MIWI2 deficiency causes more defects in addition to TE derepression. Although previous reports documented a correlation between MIWI2 inactivation and activation of TEs, ${ }^{16}$ other changes in transcriptome, genome and epigenome in Miwi2-null PGCs/prospermatogonia have not been investigated. Therefore, factors in addition to TE activation that contribute to the zygotene arrest phenotype remain unexplored. Our data presented here indicate that in spite of the known failure in DNA methylation in TE loci and at least one imprinted locus, ${ }^{46}$ Miwi2-null spermatogonia and early (leptotene and zygotene) spermatocytes all display abnormal activating and suppressive histone marks, suggesting Miwi2 inactivation also affects histone modifications in the developing male germ line. These results are consistent with a recent report demonstrating a crucial role of $\mathrm{H} 3 \mathrm{~K} 9 \mathrm{me} 2$ in silencing LINE1 in early meiotic germ cells. ${ }^{33}$ Given these changes, it is not surprising to observe significant changes in mRNA transcriptome in Miwi2-null leptotene and early zygotene spermatocytes. Downregulation of genes responsible for the transcriptional program of meiotic cell cycle progression is consistent with the early meiotic prophase arrest phenotype although the transcriptomic changes may represent secondary effects of Miwi2 inactivation. However, the meiotic defects are most likely reflective of effects secondary to persistent TE activation, a primary defect caused by Miwi2 inactivation because MIWI2 is exclusively expressed in PGCs and prospermatogonia. Moreover, the exclusive nuclear localization of MIWI2 suggests that MIWI2 mostly functions in the nucleus and its nuclear action is independent of its endonuclease activity because homozygous mutant male mice with mutation of the DDH catalytic triad of the PIWI domain are fertile. ${ }^{19}$ Another intriguing finding is that MIWI2-asscoaited piRNAs appear to be able to target mRNAs by annealing to $3^{\prime}$ UTRs. Although direct interactions between MIWI2-associated piRNAs and mRNAs remain hypothetical, this mode of action may be adopted by those non-repeat-associated piRNAs, the functions of which remain unknown. Taken together, our data suggest that many factors, including TE activation, aberrant histone modifications and altered mRNA transcriptome, contribute 
to the zygotene to pachytene arrest phenotype in Miwi2-null male mice, although the most likely primary cause lies in extensive TE transposition caused by persistent TE activation during PGC reprograming in the fetal testes.

In summary, this study provides physiological evidence supporting that MIWI2 is exclusively expressed in PGCs and prospermatogonia in fetal and early postnatal testes, and that MIWI2 is required for proper PGC reprograming, but is dispensable in postnatal testes. MIWI2 deficiencyinduced TE activation is compatible with mitotic cell cycle progression in spermatogonial development during the first wave of spermatogenesis, but can trigger the subsequent meiotic checkpoint and cause meiotic cell cycle arrests. Miwi2 cKO mice represent a good model for studying effects of TE activation on the genome of the male germ line.

\section{Materials and Methods}

Mice. Transgenic mouse lines, including Stra8-Cre, Ddx4-Cre, Prm-Cre, Ella-Cre and Mov1011/fll, were all purchased from the Jackson Laboratory (Bar Harbor, ME, USA). Breeding strategies for mice with different genotypes are depicted in Supplementary Figure S2. All mouse colonies were maintained in the animal facility of the University of Nevada, Reno, NV, USA with free access to food and water under a 12-h light and 12-h dark cycle. The Institutional Animal Care and Use Committee of the University of Nevada, Reno, NV, USA approved the mouse-use protocol.

Generation of conditional Miwi2 knockout mice. A floxed Miwi2 allele $\left(\right.$ Miwi2 $\left.^{\text {lox }}\right)$ was generated by conventional ES cell targeting through homologous recombination. ${ }^{51}$ A conditional Miwi2-targeting vector was constructed using Quick \& Easy Red/ET Conditional Knock Out Kit-loxP kit (GeneBridge, Cat\#: K005) according to the manufacturer's protocol. Briefly, two loxp site-containing PGK donor cassettes (loxP-pgk-Neor-loxP) were inserted into introns 8 and 12 of Miwi2 gene contained in a BAC clone (BAC\#:RP23-340G24, Children's Hospital Oakland Research Institute, Oakland, CA, USA), and the first PGK cassette was deleted by a Cre-expressing vector fragment (706-Cre) to retain a 34-bp loxp site sequence in the targeting vector (Figure 1a). Linearized Miwi2-targeting vector DNA $(50 \mu \mathrm{g})$ was then electroporated into mouse ES cells for homologous recombination-based gene targeting. Positive ES clones were identified using Southern blots and two external probes (for sequences, see Supplementary Table S3). The ES cell targeting efficiency was 6\%, with 12 out of 192 ES cell clones screened being heterozygous (Miwi2 ${ }^{+110 x}$ ) (Figure 1b). Two independent-targeted ES clones were injected into C57BL/6J blastocysts to produce chimeras. Male chimera mice with high-agouti percentage were bred with wild-type C57BL/6J female mice to obtain Miwi2 ${ }^{+/ 10 x}$ mice.

Histology. Hematoxylin-Eosin (HE) staining procedure for paraffin-embedded sections were performed as described before. ${ }^{41}$ For immunohistochemistry, GCNA antibody (a kind gift from Dr. Enders Mark) was used at a dilution of 1:10, and all steps were performed according to the protocol provided in the commercial $A B C$ kit (Vector Laboratory, Burlingame, CA, USA). For quantitative analyses of GCNApositive germ cells, only round or nearly round cross sections of seminiferous tubules were selected for counting and a total of at least 20 cross sections were counted for each time point.

Immunofluorescent staining. All immunofluorescent staining procedures were carried out essentially as described previously. ${ }^{52}$ The rabbit polyclonal anti-mouse MIWI2 antibodies were generated in a company (GenScript, Piscataway, NJ, USA) by immunizing rabbits with a synthetic MIWI2 peptide ( $\mathrm{N}^{\prime}$-GDGGRTFMERRGKGC-C') conjugated with a KLH adapter. The antibody dilutions used in this study were as follows: MIWI2: 1:500 dilution; ORF1: $1: 1000$ dilution (Rabbit, a kind gift from Dr. Alex Bortvin); IAP: 1:500 dilution (Rabbit, a kind gift from Dr. Bryan Cullen); $\gamma \mathrm{H} 2 \mathrm{AX}$ : 1:1000 dilution (Rabbit, Abcam, Cambridge, MA, USA).

Immunofluorescent staining on meiotic chromosome spreads was performed as described. ${ }^{53,54}$ Briefly, decapsulated seminiferous tubules were gently dispersed into single-cell suspension using two fine forceps in DMEM medium on ice. Large chunks of tubules were removed by centrifugation at $500 \times g$ for $5 \mathrm{~min}$ at room temperature. The single cells were then collected and placed in a hypotonic extraction buffer (HEB, $30 \mathrm{mM}$ Tris, $\mathrm{pH} 8.2 ; 50 \mathrm{mM}$ Sucrose; $17 \mathrm{mM}$ tri-sodium citrate; $5 \mathrm{mM}$ EDTA and $5 \mathrm{mM}$ DTT) for $10 \mathrm{~min}$. After re-suspension in $100 \mathrm{mM}$ sucrose, the single cells were spread onto slides covered by fixative buffer (1\% PFA containing $0.15 \%$ Triton X-100) and were then kept in a humidified box until use. Next, the slides were washed in $0.08 \%$ photoflow (Kodak) and air-dried at RT for $1-2 \mathrm{~h}$. The following antibodies were utilized for chromosome spreads: H3K9me3 (Millipore, Billerica, MA, USA, 1:500 dilution), H3K4me3 (Millipore, 1:500 dilution), H3K27me3 (Millipore, 1:500 dilution), SCP3 (Abcam, 1: 1000 dilution). All the final confocal images were acquired on an Olympus FV 1000 microscope with same parameters settings. Specific stages of meiotic prophase I were determined based on the criteria as previously described. ${ }^{55}$ Testes samples used for chromosome spreads were all from P21 mice unless otherwise stated.

Germ cell purification by FACS. Testes from wild type or Miwi2 cKO (Ddx4-Cre; Miwi2 ${ }^{\text {lox/ }}$ ) mice at postnatal day 10 (P10) were dissected, followed by removal of tunica albuginea with a pair of sharp tweezers under a dissection microscope. Testicular single-cell suspension was prepared by sequential digestion with Collagenase $(50 \mathrm{mg} / \mathrm{ml})$ and Trypsin $(25 \mathrm{mg} / \mathrm{ml})$ at $32^{\circ} \mathrm{C}$ for 20 min each with gently shaking. Single cells were then washed with $0.5 \% \mathrm{BSA}$ in the EKRB buffer $\left(120 \mathrm{mM} \mathrm{NaCl}, 2 \mathrm{mM} \mathrm{KCl}, 1.7 \mathrm{mM} \mathrm{CaCl}, 25 \mathrm{mM} \mathrm{NaHCO}_{3}\right.$, $1.2 \mathrm{mM} \mathrm{MgSO}_{4}, 5.6$ D-glucose, $18.5 \mathrm{mM}$ sucrose, $1.1 \mathrm{mM}$ sodium pyruvate) for three times followed by filtering through a cell strainer with the pore size of $50 \mu \mathrm{m}$ (BD Bioscience, CA, USA) before loading into a FACS sorter (Becton Dickinson, FACSAria IITM 131) using an ultra-violet laser ( $355 \mathrm{~nm}$ ) and Hoechst 33342 dye emission detector (450/50 nm BP). Sorting was performed using a $70 \mu \mathrm{m}$ nozzle at a sheath pressure of 70 p.s.i. Live cells were pregated on exclusion of propidium iodide (PI) dye (Supplementary Figure S5).

Total RNA isolation and quantitative PCR (qPCR). Total RNA was extracted from whole testes or purified cells using Trizol reagent (Invitrogen) as described previously. ${ }^{56}$ Quantitative PCR (qPCR) was performed on a 7900HT Fast Real-Time PCR system using samples in triplicates, and data were analyzed using SDS 2.4 software (Applied Biosystems, CA, USA). A reaction volume of $20 \mu \mathrm{l}$ consisted of 10 ul of $2 X$ SYBR Green PCR Master Mix (Applied Biosystems, CA, USA), 10 pmol of forward and reverse primers and $50 \mathrm{ng}$ CDNA. The PCR conditions were as follows: 1 cycle at $50^{\circ} \mathrm{C} / 2 \mathrm{~min}, 1$ cycle at $95^{\circ} \mathrm{C} / 10 \mathrm{~min}, 40$ cycles of amplification $\left(95^{\circ} \mathrm{C} / 15 \mathrm{~s}\right.$ followed by $\left.60^{\circ} \mathrm{C} / 1 \mathrm{~min}\right)$. A dissociation curve ${ }^{\Delta \Delta} \mathrm{Ct}$ analysis was conducted using SDS RQ Manager software. GAPDH was used as the internal control. The sequences of primers can be found in Supplementary Table S3.

Microarray. RNA purity was examined by measuring A260/280 ratios using a spectrophotometer (NanoDrop 2000, Wilmington, DE, USA) and RNA quality was analyzed using the Bioanalyzer (Agilent Technologies, Santa Clara, CA). Biological triplicates of purified cells were prepared from WT and Miwi2 cKO testes; each sample was derived from a pool of at least six P10 mice testes. These RNA samples were then subjected to labeling, hybridization, washing, data acquisition and analyses as described previously. ${ }^{57}$

Bioinformatic analysis. For piRNA-mRNA mapping, coding sequences (CDS), $3^{\prime}$ UTRs, and $5^{\prime}$ UTRs for all up or downregulated mRNAs with $P<0.05$ and fold change $>1.2$ (a total of 2161 genes) and 3290 randomly chosen mRNAs determined to be unregulated by MIWI2 in our microarray analyses were collected from ENSEMBL database (release 71$).^{58}$ MIWI2-associated piRNA sequences collected in a dataset containing two biological replicates were downloaded from ArrayExpress (accession number E-MTAB-730). ${ }^{19}$ MIWI2-piRNAs aligned to miRNA precursor hairpins (with 1-2 nucleotide mismatches) were excluded from further analysis. All remaining sequences that were $23-35 n$ in length and present in both biological replicates in the dataset (accession number E-MTAB-730) were considered MIWI2-associated piRNAs. Reverse complementary alignment of MIWI2-associated piRNAs against CDS, 3'UTRs and 5'UTRs was performed using our in-house software Sequery v1.0 with 1-2 mismatches allowed. ${ }^{45}$ mRNAs aligned were considered potential targets of the corresponding MIWI2-piRNAs.

\section{Conflict of Interest}

The authors declare no conflict of interest. 
Acknowledgements. We would like to thank Dr. Daniel Oliver for critical reading of this manuscript. We also thank Dr. Enders Mark, Dr. Alex Bortvin and Dr Bryan Cullen for providing the GCNA, ORF1 and IAP antibodies, respectively. This work was supported, in part, by NIH grants (HD060858, HD071736 and HD074573) to WY. All knockout mouse lines were generated and/or maintained in the University of Nevada Genetic Engineering Center (UNGEC) supported by a NIH COBRE grant (P20-RR18751).

\section{Author contributions}

Conceived and designed the experiments: JB WY. Performed the experiments: JB YZ AS. Analyzed the microarray: EN MS NO. Interpreted the data: JB WY. Wrote the manuscript: JB WY.

1. Hutvagner G, Simard MJ. Argonaute proteins: key players in RNA silencing. Nat Rev. Mol Cell Biol 2008; 9: 22-32.

2. Hock J, Meister G. The Argonaute protein family. Genome Biol 2008; 9: 210.

3. Cox DN, Chao A, Baker J, Chang L, Qiao D, Lin H. A novel class of evolutionarily conserved genes defined by piwi are essential for stem cell self-renewal. Genes Dev 1998; 12: $3715-3727$.

4. Girard A, Sachidanandam R, Hannon GJ, Carmell MA. A germline-specific class of smal RNAs binds mammalian Piwi proteins. Nature 2006; 442: 199-202.

5. Lau NC, Seto AG, Kim J, Kuramochi-Miyagawa S, Nakano T, Bartel DP et al. Characterization of the piRNA complex from rat testes. Science 2006; 313 363-367.

6. Aravin AA, Hannon GJ, Brennecke J. The Piwi-piRNA pathway provides an adaptive defense in the transposon arms race. Science 2007; 318: 761-764.

7. Aravin AA, Sachidanandam R, Girard A, Fejes-Toth K, Hannon GJ. Developmentally regulated piRNA clusters implicate MILI in transposon control. Science 2007; 316: 744-747.

8. Beyret $\mathrm{E}$, Liu N, Lin H. piRNA biogenesis during adult spermatogenesis in mice is independent of the ping-pong mechanism. Cell Res 2012; 22: 1429-1439.

9. Vourekas A, Zheng Q, Alexiou P, Maragkakis M, Kirino $Y$, Gregory BD et al. Mili and Miwi target RNA repertoire reveals piRNA biogenesis and function of Miwi in spermiogenesis. Nat Struct Mol Biol 2012; 19: 773-781.

10. Wu Q, Song R, Ortogero $\mathrm{N}$, Zheng $\mathrm{H}$, Evanoff $\mathrm{R}$, Small $\mathrm{CL}$ et al. The RNase III enzyme DROSHA is essential for microRNA production and spermatogenesis. J Biol Chem 2012; 287: 25173-25190.

11. Aravin AA, Sachidanandam R, Bourc'his D, Schaefer C, Pezic D, Toth KF et al. A piRNA pathway primed by individual transposons is linked to de novo DNA methylation in mice. Mol Cell 2008; 31: 785-799.

12. Aravin AA, van der Heijden GW, Castaneda J, Vagin VV, Hannon GJ, Bortvin A. Cytoplasmic compartmentalization of the fetal piRNA pathway in mice. PloS Genet 2009; 5 e1000764.

13. Malone CD, Brennecke J, Dus M, Stark A, McCombie WR, Sachidanandam R et al Specialized piRNA pathways act in germline and somatic tissues of the Drosophila ovary Cell 2009; 137: 522-535.

14. Huang $\mathrm{H}$, Gao Q, Peng X, Choi SY, Sarma K, Ren $\mathrm{H}$ et al. piRNA-associated germline nuage formation and spermatogenesis require MITOPLD profusogenic mitochondrialsurface lipid signaling. Dev Cell 2011; 20: 376-387.

15. Watanabe T, Chuma S, Yamamoto Y, Kuramochi-Miyagawa S, Totoki Y, Toyoda A et al. MITOPLD is a mitochondrial protein essential for nuage formation and piRNA biogenesis in the mouse germline. Dev Cell 2011; 20: 364-375.

16. Frost RJ, Hamra FK, Richardson JA, Qi X, Bassel-Duby R, Olson EN. MOV10L1 is necessary for protection of spermatocytes against retrotransposons by Piwi-interacting RNAs. Proc Natl Acad Sci USA 2010; 107: 11847-11852.

17. Zheng K, Xiol J, Reuter M, Eckardt S, Leu NA, McLaughlin KJ et al. Mouse MOV10L1 associates with Piwi proteins and is an essential component of the Piwi-interacting RNA (piRNA) pathway. Proc Natl Acad Sci USA 2010; 107: 11841-11846.

18. Zheng K, Wang PJ. Blockade of pachytene piRNA biogenesis reveals a nove requirement for maintaining post-meiotic germline genome integrity. PLOS Genet 2012 8. 1003038 .

19. De Fazio S, Bartonicek N, Di Giacomo M, Abreu-Goodger C, Sankar A, Funaya C et al. The endonuclease activity of Mili fuels piRNA amplification that silences LINE1 elements. Nature 2011; 480: 259-263.

20. Reuter M, Chuma S, Tanaka T, Franz T, Stark A, Pillai RS. Loss of the Mili-interacting Tudor domain-containing protein-1 activates transposons and alters the Mili-associated small RNA profile. Nat Struct Mol Biol 2009; 16: 639-646.

21. Shoji M, Tanaka T, Hosokawa M, Reuter M, Stark A, Kato Y et al. The TDRD9-MIWI2 complex is essential for piRNA-mediated retrotransposon silencing in the mouse male germline. Dev Cell 2009; 17: 775-787.

22. Lim AK, Tao L, Kai T. piRNAs mediate posttranscriptional retroelement silencing and localization to pi-bodies in the Drosophila germline. J Cell Biol 2009; 186 333-342.
23. Kotelnikov RN, Klenov MS, Rozovsky YM, Olenina LV, Kibanov MV, Gvozdev VA Peculiarities of piRNA-mediated post-transcriptional silencing of Stellate repeats in testes of Drosophila melanogaster. Nucleic Acids Res 2009; 37: 3254-3263.

24. Kuramochi-Miyagawa S, Watanabe T, Gotoh K, Totoki Y, Toyoda A, Ikawa M et al. DNA methylation of retrotransposon genes is regulated by Piwi family members MILI and MIWI2 in murine fetal testes. Genes Dev 2008; 22: 908-917.

25. Rouget C, Papin C, Boureux A, Meunier AC, Franco B, Robine N et al. Maternal mRNA deadenylation and decay by the piRNA pathway in the early Drosophila embryo. Nature 2010; 467: 1128-1132.

26. Zamore PD. Somatic piRNA biogenesis. EMBO J 2010; 29: 3219-3221.

27. Xiol J, Cora E, Koglgruber R, Chuma S, Subramanian S, Hosokawa M et al. A role for Fkbp6 and the chaperone machinery in piRNA amplification and transposon silencing. Mol Cell 2012; 47: 970-979.

28. Kuramochi-Miyagawa S, Kimura T, Yomogida K, Kuroiwa A, Tadokoro Y, Fujita Y et al. Two mouse piwi-related genes: miwi and mili. Mech Dev 2001; 108: 121-133.

29. Grivna ST, Pyhtila B, Lin H. MIWI associates with translational machinery and PIWI-interacting RNAs (piRNAs) in regulating spermatogenesis. Proc Natl Acad Sci USA 2006; 103: 13415-13420.

30. Carmell MA, Girard A, van de Kant HJ, Bourc'his D, Bestor TH, de Rooij DG et al. MIWI2 is essential for spermatogenesis and repression of transposons in the mouse male germline. Dev Cell 2007; 12: 503-514

31. Thomson T, Lin $\mathrm{H}$. The biogenesis and function of PIWI proteins and piRNAs: progress and prospect. Ann Rev CellDev Biol 2009; 25: 355-376.

32. Wang J, Saxe JP, Tanaka T, Chuma S, Lin H. Mili interacts with tudor domain-containing protein 1 in regulating spermatogenesis. Curr Biol 2009; 19: 640-644.

33. Di Giacomo M, Comazzetto S, Saini H, De Fazio S, Carrieri C, Morgan M et al. Multiple epigenetic mechanisms and the piRNA pathway enforce LINE1 silencing during adult spermatogenesis. Mol Cell 2013; 50: 601-608.

34. Li XZ, Roy CK, Dong X, Bolcun-Filas E, Wang J, Han BW et al. An ancient transcription factor initiates the burst of piRNA production during early meiosis in mouse testes. Mol Cell 2013; 50: 67-81.

35. Reuter M, Berninger $\mathrm{P}$, Chuma S, Shah $\mathrm{H}$, Hosokawa M, Funaya $\mathrm{C}$ et al. Miwi catalysis is required for piRNA amplification-independent LINE1 transposon silencing. Nature 2011; 480: 264-267.

36. Pillai RS, Chuma S. piRNAs and their involvement in male germline development in mice. Dev Growth Differ 2012; 54: 78-92.

37. Bao J, Yan W. Male germline control of transposable elements. Biol Reprod 2012; 865 $1-14$

38. Belgnaoui SM, Gosden RG, Semmes OJ, Haoudi A. Human LINE-1 retrotransposon induces DNA damage and apoptosis in cancer cells. Cancer Cell Int 2006; 6: 13.

39. Gallardo T, Shirley L, John GB, Castrillon DH. Generation of a germ cell-specific mouse transgenic Cre line, Vasa-Cre. Genesis 2007; 45: 413-417.

40. Sadate-Ngatchou PI, Payne CJ, Dearth AT, Braun RE. Cre recombinase activity specific to postnatal, premeiotic male germ cells in transgenic mice. Genesis 2008; 46: 738-742.

41. Bao J, Ma HY, Schuster A, Lin YM, Yan W. Incomplete cre-mediated excision leads to phenotypic differences between Stra8-iCre; Mov1011 and Stra8-iCre; Mov10l1 mice. Genesis 2013; 1-10.

42. O'Gorman S, Dagenais NA, Qian M, Marchuk Y. Protamine-Cre recombinase transgenes efficiently recombine target sequences in the male germ line of mice, but not in embryonic stem cells. Proc Natl Acad Sci USA 1997; 94: 14602-14607.

43. Blanco-Rodriguez J. GammaH2AX marks the main events of the spermatogenic process. Micros Res Tech 2009; 72: 823-832.

44. Enders GC, May JJ 2nd. Developmentally regulated expression of a mouse germ cell nuclear antigen examined from embryonic day 11 to adult in male and female mice. Dev Biol 1994; 163: 331-340.

45. Ortogero N, Hennig GW, Langille C, Ro S, McCarrey JR, Yan W. Computer-assisted annotation of murine Sertoli cell small RNA transcriptome. Biol Reprod 2013; 88: 3.

46. Watanabe T, Tomizawa S, Mitsuya K, Totoki Y, Yamamoto Y, Kuramochi-Miyagawa S et al. Role for piRNAs and noncoding RNA in de novo DNA methylation of the imprinted mouse Rasgrf1 locus. Science 2011; 332: 848-852.

47. Hayashi K, Yoshida K, Matsui Y. A histone H3 methyltransferase controls epigenetic events required for meiotic prophase. Nature 2005; 438: 374-378.

48. Tachibana M, Nozaki M, Takeda N, Shinkai Y. Functional dynamics of H3K9 methylation during meiotic prophase progression. EMBO J 2007; 26: 3346-3359.

49. Getun IV, Torres B, Bois PR. Flow cytometryurification of mouse meiotic cells. J Vis Exp 2011; 50: pii 2602

50. Huang da W, Sherman BT, Lempicki RA. Systematic and integrative analysis of large gene lists using DAVID bioinformatics resources. Nat Protoc 2009; 4: 44-57.

51. Zheng H, Stratton CJ, Morozumi K, Jin J, Yanagimachi R, Yan W. Lack of Spem1 causes aberrant cytoplasm removal, sperm deformation, and male infertility. Proc Natl Acad Sci USA 2007; 104: 6852-6857.

52. Bao J, Wang L, Lei J, Hu Y, Liu Y, Shen $\mathrm{H}$ et al. STK31(TDRD8) is dynamically regulated throughout mouse spermatogenesis and interacts with MIWI protein. Histochem Cell Biol 2012; 137: 377-389. 
53. Bao J, Wu Q, Song R, Jie Z, Zheng H, Xu C et al. RANBP17 is localized to the XY body of spermatocytes and interacts with SPEM1 on the manchette of elongating spermatids. Mol Cell Endocrinol 2011; 333: 134-142.

54. Soper SF, van der Heijden GW, Hardiman TC, Goodheart M, Martin SL, de Boer P et al. Mouse maelstrom, a component of nuage, is essential for spermatogenesis and transposon repression in meiosis. Dev Cell 2008; 15: 285-297.

55. Mahadevaiah SK, Turner JM, Baudat F, Rogakou EP, de Boer P, Blanco-Rodriguez J et al. Recombinational DNA double-strand breaks in mice precede synapsis. Nat Genet 2001; 27: 271-276.
56. Bao J, Li D, Wang L, Wu J, Hu Y, Wang Z et al. MicroRNA-449 and microRNA-34b/c function redundantly in murine testes by targeting E2F transcription factor-retinoblastoma protein (E2F-pRb) pathway. J Biol Chem 2012; 287: 21686-21698.

57. Skinner MK, Mohan M, Haque MM, Zhang B, Savenkova MI. Epigenetic transgenerational inheritance of somatic transcriptomes and epigenetic control regions. Genome Biol 2012; 13: R91.

58. Flicek P, Amode MR, Barrell D, Beal K, Brent S, Carvalho-Silva D et al. Ensembl 2012. Nucleic Acids Res 2012; 40, Database issue D84-D90.

Supplementary Information accompanies this paper on Cell Death and Differentiation website (http://www.nature.com/cdd) 